Shareholder of: Riptide Bioscience, Jesse Jaynes Shareholder of: Riptide Bioscience, James Stanway: None declared, Bahja Ahmed Abdi: None declared, Nikita Arumalla: None declared, Sarah Karrar: None declared, Sian Morris: None declared, Christopher Denton Grant/research support from: GlaxoSmithKline, Inventiva, CSF Behring, Consultant for: Roche-Genentech, Actelion, GlaxoSmithKline, Sanofi Aventis, Inventiva, CSL Behring, Boehringer Ingelheim, Bayer, David Abraham: None declared, Xu Shiwen: None declared, Richard Stratton: None declared

DOI: 10.1136/annrheumdis-2019-eular.4137

\section{OP0183 EFFICACY AND SAFETY OF RIOCIGUAT IN PATIENTS WITH EARLY DIFFUSE CUTANEOUS SYSTEMIC SCLEROSIS AND INTERSTITIAL LUNG DISEASE (SSC- ILD): RESULTS FROM THE PHASE IIB RISE-SSC STUDY}

Oliver Distler ${ }^{1}$, Yannick Allanore ${ }^{2}$, Christopher Denton ${ }^{3}$, Masataka Kuwana ${ }^{4}$, Marco Matucci-Cerinic ${ }^{5}$, Janet Pope ${ }^{6}$, Janethe de Oliveria Pena ${ }^{7}$, Kaisa Laapas ${ }^{8}$, Zhen $\mathrm{Yao}^{9}$, Friederike Behmenburg ${ }^{10}$, Melanie Hemmrich ${ }^{10}$, Dinesh Khanna ${ }^{11}$. ${ }^{1}$ University Hospital Zurich, Zurich, Switzerland; ${ }^{2}$ Cochin Hospital, Paris, France; ${ }^{3}$ University College London, London, United Kingdom; ${ }^{4}$ Nippon Medical School, Tokyo, Japan; ${ }^{5}$ University of Florence, Florence, Italy; ${ }^{6}$ University of Western Ontario, Ontario, Canada; ${ }^{7}$ Bayer US LLC, Whippany, NJ, United States of America; ${ }^{8}$ StatFinn Oy, Espoo, Finland; ${ }^{9}$ Bayer Healthcare Co., Beijing, China; ${ }^{10}$ Bayer AG, Wuppertal, Germany; ${ }^{11}$ University of Michigan, Ann Arbor, MI, United States of America

Background: Diffuse cutaneous systemic sclerosis (dcSSc) is an autoimmune connective tissue disease characterised by fibrosis and, in some patients, interstitial lung disease (ILD). Riociguat is a soluble guanylate cyclase (sGC) stimulator with vasoactive, anti-proliferative and anti-fibrotic effects. We hypothesised that riociguat may preserve lung function in patients with dcSSc.

Objectives: To evaluate the effect of riociguat on lung function in patients with early dcSSc from the multicentre, randomised, double-blind, placebo-controlled Phase Ilb RISE-SSc study (NCT02283762).

Methods: Patients had dcSSc of duration $\leq 18$ months, modified Rodnan skin score (mRSS) 10-22 units, forced vital capacity (FVC) $\geq 45 \%$ predicted (\%pred) and lung diffusion capacity for carbon monoxide (DLCO) $\geq 40 \%$ pred at screening Patients were randomised to riociguat $(n=60)$ or placebo $(n=61)$ in individually adjusted doses of $0.5 \mathrm{mg}$ up to $2.5 \mathrm{mg}$ tid. The primary endpoint was the change in mRSS from baseline to Week 52. Secondary endpoints included change in FVC\%pred from baseline to Week 52 . Effects on lung function (FVC\%pred) were assessed in the overall population and in post-hoc analyses in patients with FVC $\%$ pred $50-75 \%$ or medical history of ILD at baseline. Between groups, differences were in least-squares [LS] means.

Results: Although the study did not meet its primary endpoint, there was a reduction in $\mathrm{mRSS}$ with riociguat vs placebo $(-2.34 ; 95 \% \mathrm{Cl}:-4.99$ to $0.30 ; \mathrm{p}=0.08)$. There were no significant differences between treatment groups in the changes in FVC\%pred $(-0.20 ; 95 \% \mathrm{Cl},-3.40$ to $+3.00 ; \mathrm{p}=0.90)$ or DLCO $\%$ pred $(2.01 ; 95 \%$ $\mathrm{Cl}:-4.99$ to $6.25 ; \mathrm{p}=0.35$ ) which included all patients irrespective of the presence of SSc-ILD. However, differences between riociguat and placebo were observed when patients with SSc-ILD were analysed: At baseline, 11/60 (18\%) patients in the riociguat group and $12 / 61(20 \%)$ in the placebo group had medical history sug gesting ILD (SSc-ILD); 11/60 (18\%), and 7/61 (11\%), respectively, had restrictive lung disease (FVC\%pred $50-75 \%$ ). Results in subgroups were expressed as within-group mean change (SD). In patients with SSc-ILD, the decline in FVC\% pred between baseline and Week 52 was smaller in the riociguat group (-2.7[4.0]) than the placebo group (-8.9[13.1]). The changes in DLCO\%pred were -0.11 $(0.22)$ and $+0.87(0.71)$, respectively. In patients with restrictive lung disease, FVC \%pred decreased by $-8.7(14.0)$ at Week 52 with placebo but was almost unchanged $(+0.7[5.7])$ with riociguat. The changes in DLCO\%pred were +0.42 $(0.85)$ and $-0.27(0.39)$, respectively.

Patients with SSc-ILD receiving riociguat had lower incidences of adverse events and serious adverse events [82\% $(n=9)$ and $9 \%(n=1)$ respectively] compared with placebo [92\% $(n=11)$ and $25 \%(n=3)$ respectively] with no differences between treatment groups in the incidence of respiratory adverse events. No deaths occurred in the SSc-ILD subgroup, and no new safety signals were observed.

Conclusion: These results suggest that in an exploratory analysis, riociguat may be associated with preservation of lung function and good tolerability in patients with dcSSc and evidence of ILD on medical history.

Acknowledgement: Adelphi Communications Ltd, Bollington, UK provided medical writing support.

Disclosure of Interests: Oliver Distler Grant/research support from: Prof. Distler received research funding from Actelion, Bayer, Boehringer Ingelheim and Mitsubishi Tanabe to investigate potential treatments of scleroderma and its complications, Consultant for: Prof. Distler has/had consultancy relationship within the last 3 years with Actelion, AnaMar, Bayer, Boehringer Ingelheim, ChemomAb,
espeRare foundation, Genentech/Roche, GSK, Inventiva, Italfarmaco, iQvia, Lilly, medac, Medlmmune, Mitsubishi Tanabe Pharma, Pharmacyclics, Novartis, Pfizer, Sanofi, Serodapharm and UCB in the area of potential treatments of scleroderma and its complications. In addition, he had/has consultancy relationship within the last 3 years with A. Menarini, Amgen, Abbvie, GSK, Mepha, MSD, Pfize and UCB in the field of arthritides and related disorders, Yannick Allanore Grant research support from: Inventiva, F Hoffman La-Roche, Sanofi, BMS, Pfizer, Consultant for: Actelion, Bayer, BMS, Boehringer, Roche, Sanofi, Christopher Denton Grant/research support from: GlaxoSmithKline, Inventiva, CSF Behring, Consultant for: Roche-Genentech, Actelion, GlaxoSmithKline, Sanofi Aventis, Inventiva CSL Behring, Boehringer Ingelheim, Bayer, Masataka Kuwana Grant/research support from: Actelion, Consultant for: Chugai, Reata, GlaxoSmithKline, Bayer, Boehringer-Ingelheim, Corpus, CSL-Berling, Mochida, Speakers bureau: Actelion, Pfizer, Bayer, Nippon Shinyaku, Chugai, Marco Matucci-Cerinic Grant/ research support from: Actelion, MSD, Pfizer, BMS, Chemomab, Sanipedia Speakers bureau: Actelion, BMS; MSD, Janssen, Janet Pope Consultant for: El Lilly and Company, Janethe de Oliveria Pena Employee of: Bayer, Kaisa Laapas Employee of: StatFinn Oy, partly insourced to Bayer, Zhen Yao Employee of: Bayer, Friederike Behmenburg Employee of: Bayer AG, Melanie Hemmrich Employee of: Bayer AG, Dinesh Khanna Shareholder of: Eicos Sciences, Inc, Grant/research support from: Bayer, BMS, Pfizer, Horizon, Consultant for: Actelion Acceleron, Arena, Bayer, BI, BMS, CSL Behring, Corbus, Cytori, GSK, Genentech/Roche, Galapagos, Employee of: Elcos Sciences, Inc DOI: 10.1136/annrheumdis-2019-eular.6889

\section{OP0184 \\ PROFIBROTIC LNCRNA H19X: UNRAVELLING THE EFFECTS ON CHROMATIN REMODELING IN SYSTEMIC SCLEROSIS FIBROBLASTS}

Elena Pachera ${ }^{1}$, Shervin Assassi ${ }^{2}$, Gloria Salazar ${ }^{2}$, Mojca Frank-Bertoncelj ${ }^{1}$, Jörg Distler ${ }^{3}$, Gabriela Kania ${ }^{1}$, Oliver Distler ${ }^{1} .{ }^{1}$ Center of Experimental Rheumatology, University Hospital Zurich, Zurich, Switzerland; ${ }^{2}$ Department of Internal Medicine, University of Texas Health Science Center at Houston, Houston, United States of America; ${ }^{3}$ Department of Internal Medicine 3, University of Erlangen, Erlangen, Germany

Background: Long noncoding RNAs (IncRNAs) are noncoding transcripts involved in the regulation of gene expression in health and disease. LncRNAs function via different mechanisms either acting cis, regulating the expression of nearby genes, or in trans, modulating the expression of distant genes. We identified a novel TGF $\beta$ regulated IncRNA, H19X, which was strongly upregulated in a wide variety of fibrotic disorders. Moreover, we were able to demonstrate that $\mathrm{H} 19 \mathrm{X}$ is a key driver of myofibroblast formation and extracellular matrix (ECM) overproduction.

Objectives: To define the mode of action of H19X (cis or trans) and to assess $\mathrm{H} 19 \mathrm{X}$ function on chromatin rearrangements that may drive myofibroblast differentiation and ECM production.

Methods: The function of H19X was investigated in SSc dermal fibroblasts $(n=5)$ by knocking down $\mathrm{H} 19 \mathrm{X}$ with locked nucleic acid oligonucleotides followed by TGF- $\beta$ stimulation and microarray analysis with Illumina HT-12 arrays. In situ hybridization for $\mathrm{H} 19 \mathrm{X}$ was performed using Stellaris FISH probes. Chromatin remodeling was investigated by Assay for Transposase-Accessible Chromatin using sequencing (ATAC-seq).

Results: As analyzed by microarray, none of the 16 genes belonging to the same genomic region as $\mathrm{H} 19 \mathrm{X}$ locus was affected by its downregulation, indicating that in-cis regulation is not a major mechanism of $\mathrm{H} 19 \mathrm{X}$ activity. Cellular localization can give a first indication about IncRNA trans function: FISH staining of SSc fibroblasts stimulated with TGF- $\beta$ revealed tightly localized nuclear foci in TGF- $\beta$ treated cells. $\mathrm{H} 19 \mathrm{X}$ nuclear localization was confirmed by cell fractionation, where $\mathrm{H} 19 \mathrm{X}$ expression was peaking in the nucleus of cells after $6 \mathrm{~h}$ of treatment with TGF- $\beta$. We next hypothesized that $\mathrm{H} 19 \mathrm{X}$ nuclear expression might induce chromatin rearrangements. We assessed whether chromatin changes caused by H19X knockdown might be correlated with changes in expression profile seen by microarray. Therefore, we analyzed the differences in the chromatin conformation flanking transcription start sites (TSSs) by ATAC-seq. A clear separation between the two experimental groups (scrambled vs H19X knockdown) was observed and 450 differentially accessible regions were identified $(p<0.05$, Log2FC $>0.5)$.

After focusing on genes that displayed significant variations and Log2FC $>0.5$ for both the array and ATAC-seq analysis, eight candidates genes were found both upregulated by microarray and showed increased accessibility at their TSSs by ATAC-Seq. Similarly, three candidate genes were identified as being downregu lated by microarray and showed decreased TSS accessibility by ATAC-Seq These genes included histones HIST1H2BD and HIST1H1C, suggesting a direct regulation of chromatin organization as a mode of action for $\mathrm{H} 19 \mathrm{X}$. Moreover, the cell cycle progression inhibitors FABP3 and PTTG1 were pointing to a role of $\mathrm{H} 19 \mathrm{X}$ in proliferation. 